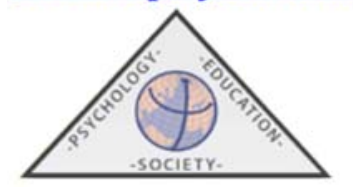

ISSN 2171-2085 (print) / ISSN 1989-709X (online)

\title{
Tipos de personalidad, agresión y conducta antisocial en adolescentes
}

\author{
Consuelo MORÁN, José A. CARMONA y José FÍNEZ
}

\author{
Universidad de León, España \\ (Received on February 3, 2016; Accepted on February 26, 2016)
}

RESUMEN: Basado en el Cuestionario de Personalidad de Eysenck para jóvenes (EPQ-J), se analizan los tipos de personalidad y su relación con la agresividad y la conducta antisocial en una muestra de estudiantes $(N=1416)$ de entre 11 y 15 años de edad (edad media $=13,32 ; D T=1,22)$. Mediante análisis de clúster se hallaron tres tipos de personalidad que se relacionaron con la hipótesis de Eysenck sobre la conducta antisocial y el nivel de agresividad evaluado mediante del Aggresion Questionnaire (AQ) de Buss y Perry (1992) en su versión reducida(Bryant y Smith (2001). El perfil del tipo infracontrolado confirmó la hipótesis de la conducta antisocial, siendo también el tipo más agresivo. Los tipos infracontrolado y supracontrolado estaban implicados en acoso escolar, aunque de manera diferente. El tipo resiliente mostró un perfil más adaptativo y mejor rendimiento académico. Ambos sexos fueron diferentes en dimensiones de personalidad y agresión. Se destaca la importancia de la agresión entre jóvenes adolescentes y la necesidad de más investigación sobre esta problemática.

Palabras clave: Tipos de personalidad, adolescencia, conducta antisocial, acoso escolar, agresividad.

\section{Personality types, aggression and antisocial behavior in adolescents}

\begin{abstract}
Based on the Junior Eysenck's Personality Questionnaire (EPQ-J), the types of personality and its relationship with aggressiveness and the antisocial behavior is analyzed in a student's sample $(N=1416)$ with ages between 11 y 15 years old (average age $=13,32 ; S D=1,22$ ). Cluster analysis using the reduced version (Bryant y Smith (2001) of the Aggression Questionnaire(AQ)(Buss y Perry, 1992) revealed three personality types that were related to Eysenck's hypothesis of antisocial behavior and the level of aggressiveness. The under controlled profile confirmed the Eysenck's hypothesis of antisocial behavior in early adolescence, and was also found to be the most aggressive prototype. The under controlled and over controlled types were implicated in bullying, but in different ways. Furthermore, the resilient people were found to have an adaptive profile combined with the best academic achievement. Gender differences were also found in personality dimensions and aggression. The importance of aggression among young adolescents and the necessity of further research on this topic are emphasized.
\end{abstract}

Keywords: Personality types; adolescence; antisocial behavior; bullying; aggressiveness.

Correspondencia: Consuelo Morán Astorga, Universidad de León. Departamento de Psicología, Sociología y Filosofía. Facultad de Educación. Campus de Vegazana, s/n. 24071 León (España). E-mail: mcmora@unileon.es 


\section{Introducción}

En el marco del maltrato entre iguales que aparece en contextos escolares, la conducta agresiva se muestra como un fenómeno persistente entre los agresores, pudiendo ser una característica estable de la personalidad (Buss, 1961). En cuanto alos distintos tipos de comportamientos agresivos, los investigadores distinguen tres clases de niños o adolescentes agresivos: los agresores proactivos, reactivos y relacionales (Griffin y Gross, 2004). La agresión proactiva la practican sujetos que están seguros de que la agresión les aporta beneficios con facilidad, que creen aumentar su autoestima al sentirse dominadores de otros, y que creen que el sometimiento de los demás se va a producir sin que ello conlleve graves daños (Crick y Dodge, 1996), como reflejan también Sánchez, Ortega y Menesini (2012) quienes concluyen que los agresores reconocen sentirse bien por lo que hacen. Por su parte, la agresión relacional puede incluir conductas o amenazas, como las frecuentes de excluir a otra persona del grupo de iguales, ignorarla o hacerle daño en las relaciones con sus compañeros a través de rumores y murmuraciones (Ortega, Elipe y Calmaestra, 2009). En definitiva, se trata de intentar dañar la autoestima de otra persona, sus amistades o estatus social (Anderson y Bushman, 2002). En un estudio con 384 adolescentes de 12-14 años, Ojanen, Findley y Fuller (2012) investigaron la agresión física y la agresión relacional en su asociación con variables de personalidad y de metas sociales. Los varones se implicaban en la agresión física por metas de dominancia y narcisismo, en tanto que en la agresión relacional se implicaban ambos sexos por metas de dominancia. La frustración y la afiliación se asociaban a la agresión relacional. Ojanen et al. (2012) concluyen que sus hallazgos apoyan y amplían la investigación anterior, al tiempo que sugieren que la personalidad y las metas sociales se hallan en estrecha asociación con la agresión física y la relacional en un contexto de iguales. En el estudio de Ramírez, Díaz y López (2015) también aparecen diferencias por género siendo los varones quienes utilizan el maltrato directo (insultos, amenazas y acoso sexual) en tanto que las mujeres utilizaban más el maltrato indirecto (e.g.: ignorar, hablar mal y difundir rumores).

Las personas que tienen dificultad con su autocontrol es poco probable que se contengan aunque sus conductas resulten antisociales y agresivas. De hecho, un fallo en el autocontrol se caracteriza por la tendencia a reaccionar a las situaciones sin haber pensado en las consecuencias posteriores. Según esto, las variables de personalidad caracterizadas por fallo en la auto-regulación es muy probable que estén asociadas a niveles altos de conducta agresiva (e.g.,Bettencourt, Talley, Benjamin y Valentine, 2006; Block y Block, 1980).

Se ha comprobado, en el estudio de los principales modelos de personalidad, que éstos tienen dimensiones asociadas a actos antisociales. En el modelo de los Big Five (Egan, 2009), los individuos bajos en A (amabilidad) y en C (responsabilidad) son potencialmente más violentos, o ya actúan con mayor violencia real, sean niños, adultos, hombres o mujeres. Se acentúa aún más la asociación de las dimensiones A y C (bajas) con agresión, si a ellas se añade $\mathrm{N}$ (neuroticismo) alto. En cambio, E (extraversión) y O (apertura a la experiencia) no están relacionadas con agresión. Con este mismo Modelo de los Cinco Factores, y edades entre 18 y 55 años, Grumm y von Collani (2009) caracterizaron al tipo de bajo autocontrol (infracontrolado) principalmente por su baja amabilidad y su baja responsabilidad; este tipo fue el que más puntuó en el Cuestionario de Agresión de Buss-Perry (1992) en esa investigación. Los individuos clasificados como infracontrolados en el estudio de Klimstra, Hale III, 
Raaijmakers, Branjey Meeus (2010), en una muestra de 923 adolescentes entre 12 y 16 años, mostraron las puntuaciones más bajas en las dimensiones A y $\mathrm{C}$ a lo largo de todas las edades de la muestra. Además, los infracontrolados eran los que presentaban los niveles más altos de delincuencia. Los adolescentes delincuentes eran en general más desinhibidos y más agresivos que los adolescentes bien adaptados (Eklund, Liljeberg y Klinteberg, 2011). Otro estudio realizado por Mendoza, Morales y Arriaga (2015) con 1263 estudiantes de bachiller del estado de Méjico concluye que los escolares que participan como acosadores manifiestan más conductas antisociales, tienen menos amigos y presentan más conductas disruptivas en el aula y más conflictos en la interacción con los profesores.

Una asociación particularmente fuerte se establece entre la dimensión P (psicoticismo) y la agresión, en el modelo de personalidad de Eysenck (Egan, 2009).Conviene recordar que para Eysenck (1992) las dimensiones amabilidad y responsabilidad del modelo de los Cinco Factores son en realidad facetas del psicoticismo.El modelo de personalidad de Eysenck se basa en la concepción de que las manifestaciones fenotípicas de la conducta reflejan rasgos subyacentes de origen biológico (Eysenck, 1997).

$\mathrm{Al}$ modelo de Eysenck se le conoce como modelo PEN, debido a sus tres dimensiones o factores de la personalidad: Psicoticismo (P), Extraversión (E) y Neuroticismo (N). Estas dimensiones de la personalidad tienen carácter continuo y son supuestamente independientes. El neuroticismo $(\mathrm{N})$ se considera que refleja la tendencia a experimentar emociones negativas, inestabilidad emocional e ideas más irracionales en el nivel cognitivo. La extraversión expresa rasgos como sociabilidad, actividad, vitalidad, asertividad, indicando las puntuaciones bajas en extraversión mayor predominio de los rasgos de introversión. El psicoticismo incluye rasgos de agresividad y de conducta antisocial. Las puntuaciones bajas en psicoticismo indican que los individuos se hallan dentro de la normalidad y que en ellos predomina el control de impulsos. Se viene informando de que la dimensión psicoticismo, en sus puntuaciones altas, predice mala adaptación y conductas antisociales y delictivas (Center, Jackson y Kemp, 2005; López y López, 2003).

La estrategia de investigar en grupos normales el comportamiento antisocial supone que se entiende éste en sentido amplio, incluyendo muchas formas del mismo de suyo menos serias que otras agresiones o transgresiones. La teoría de Eysenck predice que los individuos altos en la dimensión psicoticismo estarán predispuestos a desarrollar conducta antisocial (Eysenck, 1997).Un individuo que puntúa alto tanto en extraversión como en psicoticismo estará predispuesto a desarrollar una conducta antisocial, especialmente agresiva, ya que ésta se asocia con arousal cortical bajo. Esto es lo que ocurre en los altos en extraversión, quienes poseen un sistema nervioso escasamente reactivo y no aprenden las conductas gobernadas por reglas tan fácilmente como lo hacen los individuos con un nivel de base más alto de arousal cortical. Cuando estos individuos son también altos en neuroticismo, se añade un carácter emocional e irracional a su conducta en las mismas circunstancias (Center et al., 2005). Todavía hay una puntuación aportada por la escala S -sinceridad- (L de Lie, en inglés) en el Cuestionario de Personalidad de Eysenck para adolescentes (Eysenck y Eysenck, 1998). Los individuos antisociales típicamente puntúan más alto que los demás en insinceridad o disimulo (L). En consecuencia, la conducta antisocial vendrá asociada a puntuaciones altas en extraversión, en neuroticismo, y, sobre todo enpsicoticismo y en insinceridad. Ésta es la forma más fuerte de la hipótesis de Eysenck sobre la conducta antisocial (Center et al., 2005). 
La presente investigación, con amplia muestra de adolescentes españoles entre los 11 y 15 años de edad, se propone comprobar con niños y adolescentes normales la hipótesis de la conducta antisocial de Eysenck (Eysenck y Eysenck, 1985). Se espera validar internamente en la muestra estudiada los tres principales prototipos de la personalidad, los cuales ya vienen replicándose desde hace varios años, por ejemplo en Robins et al. (1996). Por su parte, se espera que se confirme la hipótesis de Eysenck sobre la conducta antisocial (CA) en adolescentes, dentro de su modelo biosocial PEN de la personalidad. Se espera que la variable psicoticismo (Eysenck y Eysenck, 1985) destaque en el prototipo infracontrolado. Esperamos que las diferencias entre los tres prototipos en psicoticismo se mantengan estables desde los 11 a los 15 años. Es decir, si psicoticismo es una dimensión estable o más bien varía con la edad. Por último, con respecto a la validación externa, esperamos que los ANOVAs separados para diversas variables dependientes, como las cuatro subescalas de agresión, diferencien también a los prototipos de personalidad en predisposición a conductas antisociales agresivas, así como también en conductas de adaptación escolar, en especial, en rendimiento académico.

\section{Método}

\section{Participantes}

La muestra estuvo compuesta por un total de 1416 adolescentes (658hombres -47\%- y 758 mujeres -53\%), con edades comprendidas entre los 11 y 15 años (con una media de 13,32 años y desviación típica de 1,22). De 11 años eran 96; 294 de 12; 397 de 13; 313 de 14 y 316 de 15. Todos escolarizados en cinco colegios públicos y privados de la Comunidad de Castilla y León (España). Fueron escogidos seis colegios, pero uno de ellos no deseó participar, la forma de elección fue por conveniencia (cercanía).

\section{Procedimiento y método}

Se trata de un estudio descriptivo y comparativo. Es regional y ex post facto. Se realizó a través de cuestionarios de auto-informe. Los cuestionarios se aplicaron en el contexto del aula. Previo a su administración, se contó con el permiso de los directores de los colegios y con el consentimiento de padres y tutores. Únicamente dos niños no realizaron las pruebas. Los cuestionarios fueron aplicados por personas expertas (psicólogos, psicopedagogos y tutores), siendo la duración de las sesiones de una hora aproximadamente. En todo momento se garantizó a los participantes el anonimato y la confidencialidad de los datos.

\section{Instrumentos de evaluación}

Cuestionario de Personalidad de Eysenck (EPQ-J). El Cuestionario de Personalidad EPQ (Eysenck y Eysenck, 1998) tiene la forma J aplicable a niños y adolescentes entre 8 y 15 años de edad. Consta de 81 ítems con opciones de respuesta de tipo dicotómico sí-no. Mide las dimensiones de la personalidad según el modelo de Eysenck: neuroticismo (20 ítems), extraversión (24 ítems) y psicoticismo (17 ítems); otros 20 ítems de la escala de sinceridad (S) completan el EPQ-J. Aunque los coeficientes de psicoticismo y sinceridad son relativamente bajos en fiabilidad en nuestro estudio, sin embargo están en línea con los informados porEysenck, Eysenck y Barrett (1985). 
Conducta antisocial (CA). Esta medida resulta de una selección realizada por Eysenck y Eysenck (1998) entre los anteriores ítems, selección de 35 de ellos que figuran en el manual y que aporta la quinta medición posible con el Cuestionario EPQ-J. La Conducta Antisocial es un compuesto de hasta 35 puntos posibles, es decir, que se puntúa entre un mínimo de 0 y un máximo de 35 puntos: 9 de neuroticismo, 15 de extraversión y 11 de psicoticismo. La fiabilidad para esta escala hallada por los autores de la versión española fue de 0,75 , por lo que la consideraron un instrumento útil para predecir la delincuencia o la reincidencia de los sujetos (Eysenck y Eysenck, 1998).

Cuestionario de Agresión(AQ reducido) (Bryant y Smith, 2001). Se trata de la versión reducida del Aggression Questionnaire (AQ) de Buss y Perry (1992), el cual estaba compuesto originalmente por un total de 29 ítems agrupados en 4 dimensiones. La versión reducida de Bryant y Smith (2001) -que ha sido la que hemos utilizado en el presente estudio-consta de un total de 12 ítems agrupados en 4 dimensiones: agresión física, agresión verbal, enfado y hostilidad. Las propiedades psicométricas de las escalas reducidas del Cuestionario de Agresión muestran valores altos $(\alpha=.88$ - .92) de consistencia interna en términos del alfa de Cronbach; los datos de validez convergente y discriminante proporcionaron un fuerte apoyo para esta arquitectura de la agresión para los factores agresión física, enfado y hostilidad por su fuerte correlación con la medida criterio. Sin embargo, el factor agresión verbal no mostró ninguna validez discriminante con la medida criterio y mostró baja correlación con cada uno de los otros factores del AQ (Bryant y Smith, 2001).

Escalas de adaptación escolar: Son escalas de ítem único preparadas ad hocpara el presente estudio, a las cuales se contesta con puntuaciones que oscilan entre 1 y 5 :

- $\quad$ Maltrato escolar recibido. Sentir maltrato escolar. Se le pide al estudiante que conteste cómo le han tratado el último año en el colegio, desde 1 (muy bien) a 5 (muy mal).Los niveles intermedios de respuesta son 2-3-4.

- $\quad$ Agresión relacional a iguales. Causante de maltrato escolar. De modo similar, se le pregunta si en el último año ha tratado mal a otros compañeros, para que conteste 1 (nunca) hasta 5 (muchas veces), con las respuestas intermedias de 2-3-4.

Rendimiento académico. Por último, se le pregunta acerca de cómo lleva los estudios en general, comparado con sus compañeros, pudiendo contestar desde 1 (muy mal) a 5 (muy bien), pasando por 2 (mal), 3 (regular) y 4 (bien).

\section{Análisis de datos}

Por un lado, se realizó un análisis de clúster para clasificar a los sujetos en grupos; se hicieron análisis estadísticos descriptivos y de frecuencias; la prueba $t$ de diferencia de medias para averiguar las diferencias por razón de género en las medidas aplicadas; la prueba $\chi^{2}$ se realizó para determinar si las diferencias entre los grupos (tipos) y género eran significativas; se realizaron ANOVAs para examinar la presencia de diferencias entre los grupos en las escalas aplicadas; y la prueba Newman-Keulspara estimar en qué grupos era significativa esa diferencia. Los análisis se realizaron utilizando el paquete estadístico SPSS, versión 20. 


\section{Resultados}

\section{La solución de tres conglomerados}

Los resultados del análisis de conglomerados (análisis de clúster) se muestran en la tabla 1. Hemos optado por la solución de tres conglomerados, por ser la solución preferida en la literatura sobre prototipos de personalidad y tener una sólida base teórica que apoya su interpretación. Según lo esperado, han aparecido los tres prototipos de la personalidad adolescente: el supracontrolado, el infracontrolado y el resiliente. Los porcentajes de cada sexo en los prototipos o grupos, así como su caracterización, son semejantes a los encontrados por otros autores mediante una solución también de tres conglomerados, por el método de Ward y el procedimiento de agrupación K-medias (Klimstra et al., 2010).

En la distribución del sexo en los tres conglomerados, se ha hallado diferencia significativa $\left(\chi^{2}=24,67, p<, 01\right)$. Esta significación estadística del $\chi^{2}$, superior a la exigida para el nivel de confianza del 1\% (24,67 frente a la requerida de 9,21 para 2 grados de libertad), pone de manifiesto que la distribución del sexo en los grupos no se debe al azar. Las mujeres superan en porcentaje a los varones ligeramente en el prototipo resiliente o bien adaptado (22,2\% por $17,6 \%$ en varones) y ampliamente en el prototipo supracontrolado (15,2\% por 9,4\% en varones). Ese predominio se invierte en el conglomerado infracontrolado, en el que los chicos llegan al 19,5\% frente al 16,1\% de las chicas. En conjunto, el prototipo resiliente es el más numeroso de los tres con el 39,8\% de la muestra, mientras que en el infracontrolado se clasifica el 35,6\% y en el supracontrolado lo hace el 24,6\%.

Tabla 1. Participantes por prototipos de personalidad o grupos (G) y sexo

\begin{tabular}{cccc}
\hline & & Prototipo & \\
\hline & Supracontrolado & Infracontrolado & Resiliente \\
\hline Hombres & $133(9,4 \%)$ & $276(19,5 \%)$ & $249(17,6 \%)$ \\
Mujeres & $215(15,2 \%)$ & $228(16,1 \%)$ & $315(22,2 \%)$ \\
Totales & $348(24,6 \%)$ & $504(35,6 \%)$ & $564(39,8 \%)$ \\
\hline
\end{tabular}

\section{Descripción de los prototipos de personalidad}

$\mathrm{Si}$ atendemos a los valores de las medias para cada uno de los prototipos estudiados (véase figura 1), el prototipo supracontrolado muestra un perfil de personalidad con el más alto nivel de neuroticismo, la más baja extraversión (alta introversión), estando en torno a la media en psicoticismo y sinceridad. Este grupo supracontrolado tiene valores en neuroticismo y extraversión contrapuestos a los valores de esas mismas dimensiones en el prototipo resiliente 0 mejor adaptado, por lo que a veces se le llama prototipo vulnerable. El perfil del prototipo infracontrolado muestra las puntuaciones más altas en psicoticismo y en sinceridad, estando también claramente por encima de la media en neuroticismo y extraversión. A este grupo infracontrolado podemos llamarle el prototipo agresivo, a causa de su elevado psicoticismo. En la muestra estudiada, el perfil del $40 \%$ de la población de adolescentes es del prototipo resiliente, el más numeroso y mejor adaptado, con puntuaciones muy bajas en neuroticismo, 
psicoticismo y sinceridad en tanto que altas en extraversión. Se contrapone al infracontrolado o agresivo-. Es el grupo de referencia para comprobar hasta qué punto los grupos vulnerable (supracontrolado) y agresivo (infracontrolado) se apartan en sus niveles de agresividad y formas de acoso de los comportamientos deseables y adaptativos (en las Figuras 1 y 2 se ilustran estas diferencias entre los tres perfiles de personalidad adolescente).

Plot of Means for Each Cluster

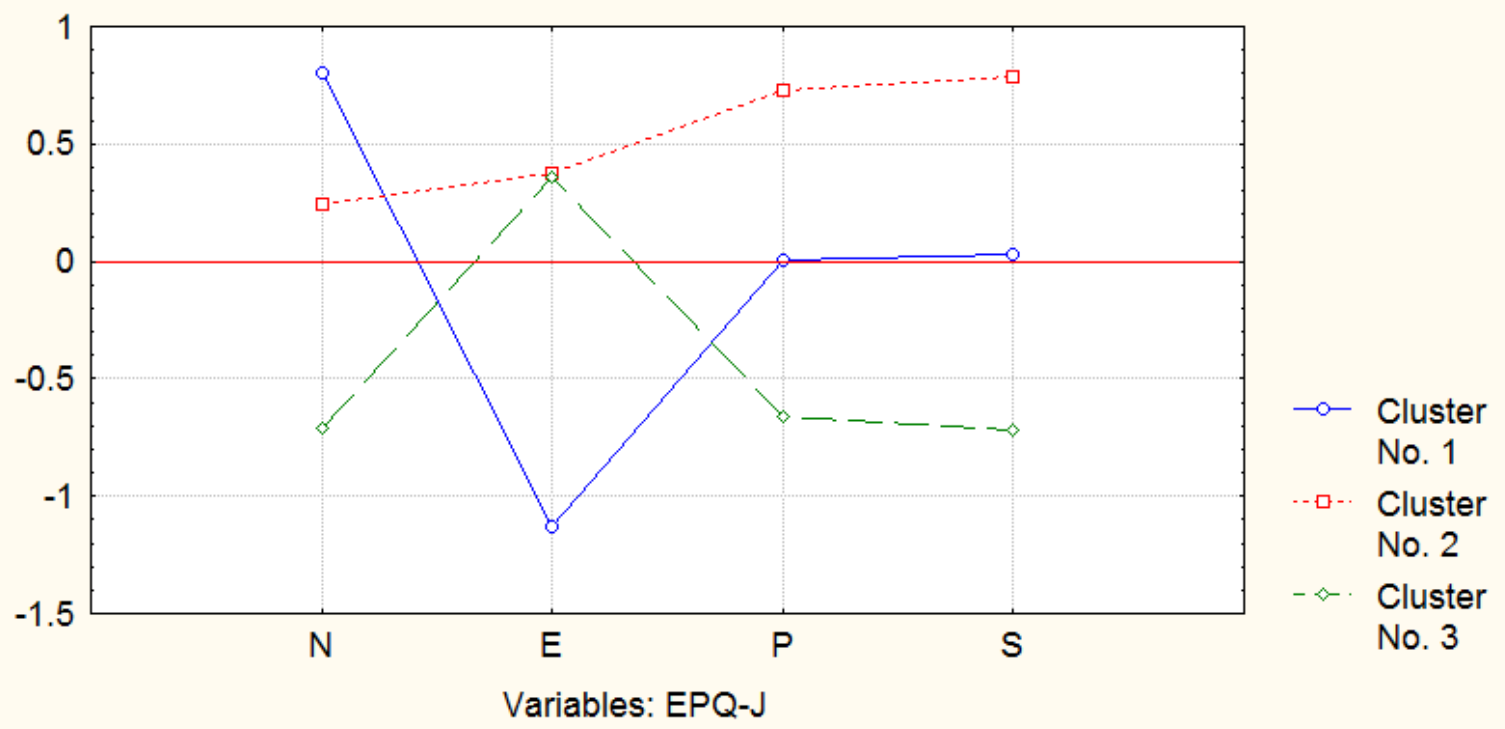

Figura 1. Representación de las medias tipificadas de los tres conglomerados en base a las puntuaciones obtenidas en el EPQ-J. Clúster 1 = supracontrolado; Clúster 2 = infracontrolado; Clúster 3 = resiliente. $\mathrm{N}=$ neuroticismo; $\mathrm{E}=$ extraversión; $\mathrm{P}=$ psicoticismo; $\mathrm{S}=$ sinceridad. $\mathrm{EPQ}-\mathrm{J}=$ Cuestionario de Personalidad de Eysenck.

Diferencias en agresión y funcionamiento escolar de los tres tipos de personalidad

La tabla 2 nos permite observar las diferencias del grupo resiliente con relación al supracontrolado y al infracontrolado, en todas las variables estudiadas, excepto en extraversión en la cual no hay diferencias significativas entre los grupos resiliente e infracontrolado. Existe una diferencia menor $(p=, 000)$ en las respuestas que dan los resilientes frente a los infracontrolados a sentirse alguna vez maltratados o acosados. Por lo demás, el prototipo de escaso control del ego, el infracontrolado, puede ser inadaptado cuando en las circunstancias se producen agresiones de cualquier clase. Los individuos clasificados en el infracontroladomuestran las puntuaciones más altas de los tres grupos en psicoticismo y en sinceridad en el EPQ-J.

Estas diferencias del infracontrolado, con relación al supracontrolado y sobre todo al resiliente, por su alto psicoticismo en el EPQ-J son estables a través de las diferentes edades estudiadas -entre los 11 y los 15 años- (véase Figura 2). Su perfil en este cuestionario apoya la hipótesis de la conducta antisocial, en cuya escala supera ampliamente $(\mathrm{p}<, 001)$ a los otros dos grupos. En validación externa con las escalas de agresión, el grupo infracontrolado puntúa muy alto en las tres primeras (física, verbal y enfado), sólo superado por el supracontrolado en 
hostilidad. Hay que destacar, en cambio, que el infracontrolado no se diferencia del supracontrolado ni en agresión verbal ni en enfado, lo que indica que tanto los infracontrolados como los supracontrolados son igualmente superiores a los resilientes en esas dos escalas de agresión, con una diferencia estadística altamente significativa $(\mathrm{p}=, 000)$.

Tabla 2. Puntuaciones medias de los grupos o prototipos de personalidad en las escalas utilizadas y resultados del análisis de varianza (ANOVA)

\begin{tabular}{lcccccl}
\hline Escalas & \multicolumn{3}{c}{ Prototipos } & & & \multicolumn{2}{l}{ ANOVA } \\
\hline EPQ-J & $\begin{array}{c}\text { Supra- } \\
\text { controlado }\end{array}$ & $\begin{array}{c}\text { Infra- } \\
\text { controlado }\end{array}$ & Resiliente & $F$ & $p$ & Newman-Keuls \\
\hline Neuroticismo & 12,92 & 10,56 & 6,57 & 449,67 &, 000 & $1>2$ y 3***; 2>3*** \\
Extraversión & 13,62 & 18,84 & 18,80 & 525,05 &, 000 & $1<2$ y 3***; $2=3$ \\
Psicoticismo & 3,55 & 5,57 & 1,69 & 414,70 &, 000 & $2>1$ y 3***; $1>3 * * *$ \\
Sinceridad & 11,72 & 14,87 & 8,58 & 513,86 &, 000 & $2>1$ y 3***; $1>3 * * *$ \\
CA & 17,60 & 21,59 & 15,91 & 390,69 &, 000 & $2>1$ y 3***; $1>3 * * *$
\end{tabular}

\begin{tabular}{|c|c|c|c|c|c|c|}
\hline Agresión & & & & & & \\
\hline Física & 6,08 & 7,73 & 4,73 & 129,99 & 000 & $2>1$ y $3^{* * *} ; 1>3^{* * *}$ \\
\hline Verbal & 7,22 & 7,45 & 5,85 & 15,66 & ,000 & $3<1$ y $2 * * * ; 1=2$ \\
\hline Enfado & 7,86 & 8,23 & 6,45 & 76,23 & ,000 & $3<1$ y $2 * * * ; 1=2$ \\
\hline Hostilidad & 9,12 & 8,16 & 6,30 & 144,11 & ,000 & $1>2$ y $3^{* * *} ; 2>3 * * *$ \\
\hline \multicolumn{7}{|l|}{ Adaptación } \\
\hline Víctima & 1,69 & 1,33 & 1,22 & 48,02 & ,000 & $1>2$ y $3 * * * ; 2>3 *$ \\
\hline Agresor & 1,34 & 1,52 & 1,15 & 44,03 & ,000 & $3<1$ y $2^{* * *} ; 1<2 * *$ \\
\hline Rendimiento & 3,08 & 3,14 & 3,44 & 29,25 & ,000 & $3>1$ y $2 * * * ; 1=2$ \\
\hline
\end{tabular}

Nota. ANOVAs para las subescalas del EPQ-J y CA (Conducta Antisocial), las 4 subescalas del Cuestionario de Agresión y las tres medidas de Adaptación Escolar: maltrato escolar recibido (víctima), agresión relacionala iguales (agresor) y rendimiento académico. Comprobación Post-Hoc, mediante la prueba de Newman-Keuls, del nivel de significación de las diferencias entre los tres Grupos o Clusters. $* * *=p<, 001 ; * *=p<, 01 ; *=p<, 05$. Supra-controlado = Cluster1; Infracontrolado = Cluster 2; Resiliente $=$ Cluster 3.

Por su parte, si atendemos a las medidas de adaptación escolar de los participantes según sean víctimas de acoso, acosadores y su rendimiento escolar, ambos grupos con alteración en el control del ego, esto es, el supracontrolado (por exceso) e infracontrolado (por defecto), muestran diferencias entre sí en cuanto al acoso recibido y la agresión perpetrada. Estando ambos por encima de los niveles de acoso y victimización mostrados por el grupo resiliente. En cuanto al rendimiento académico, al contrario de lo que ocurre con todos los tipos de agresión y conducta antisocial, los grupos supracontrolado e infracontrolado se encuentran ambos por debajo en rendimiento académico comparado con el grupo resiliente (ver tabla 2). 


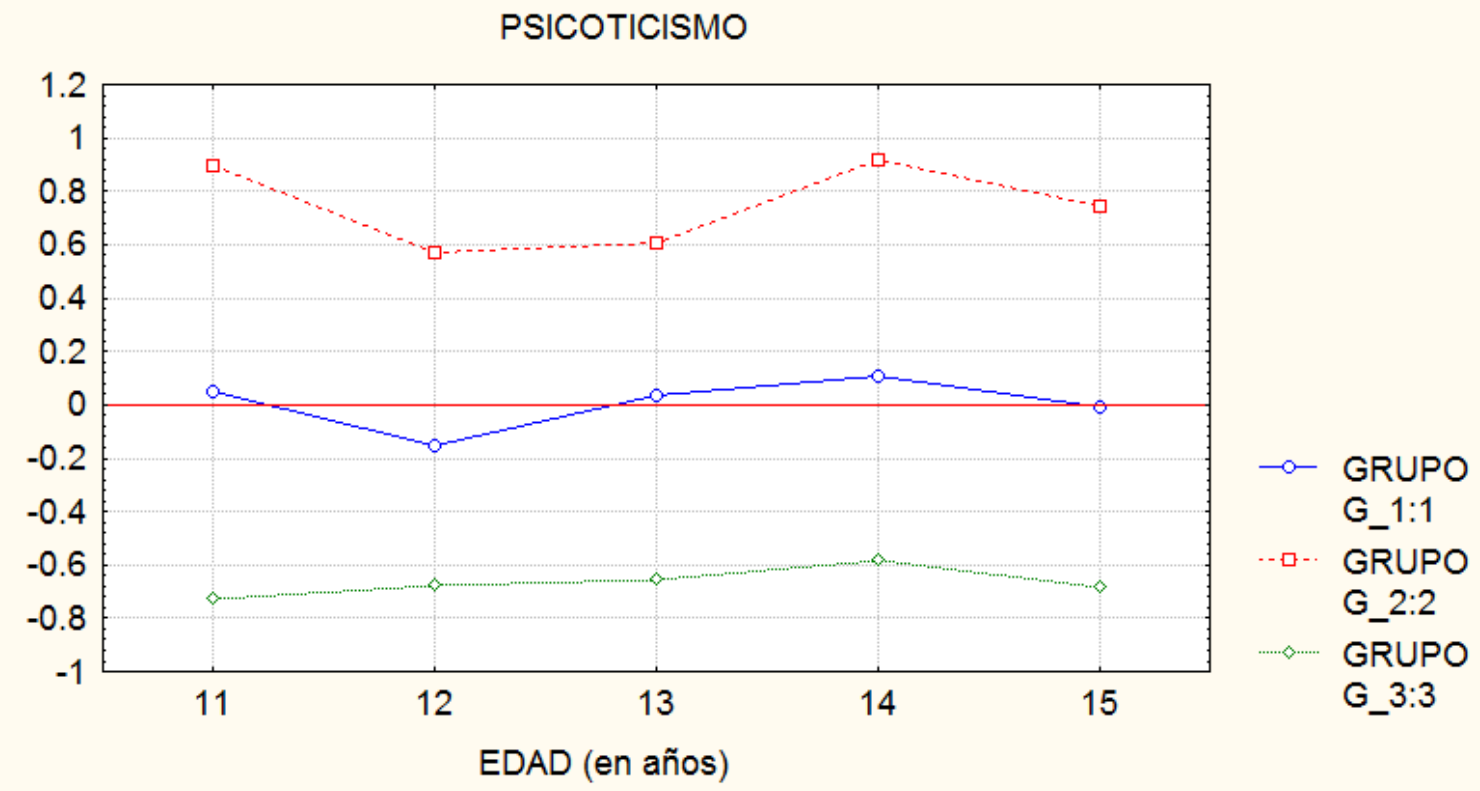

Figura 2. Representación, en puntuaciones tipificadas, de las diferencias entre los tres Grupos en la dimensión psicoticismo según edades Grupo 1 = supracontrolado; grupo 2 = infracontrolado; grupo $3=$ resiliente

Con respecto a la interacción entre el grupo de personalidad y la implicación en acoso se ha obtenido un valor de la $F$ elevada estadísticamente significativo. En concreto, el clúster grupo 1(supracontrolado) se ha mostrado más acosado y menos acosador si lo comparamos con el clúster 2 (infracontrolado), ocurriendo justo lo contrario, mostrándose menos acosado pero más acosador que el clúster 1 . Por su parte, el grupo 3 (resiliente) presenta los menores niveles de acoso y victimización, lo que indicaría que no está implicado en la agresión relacional, no estando implicado ni como acosado ni como acosador (ver la Figura 3).

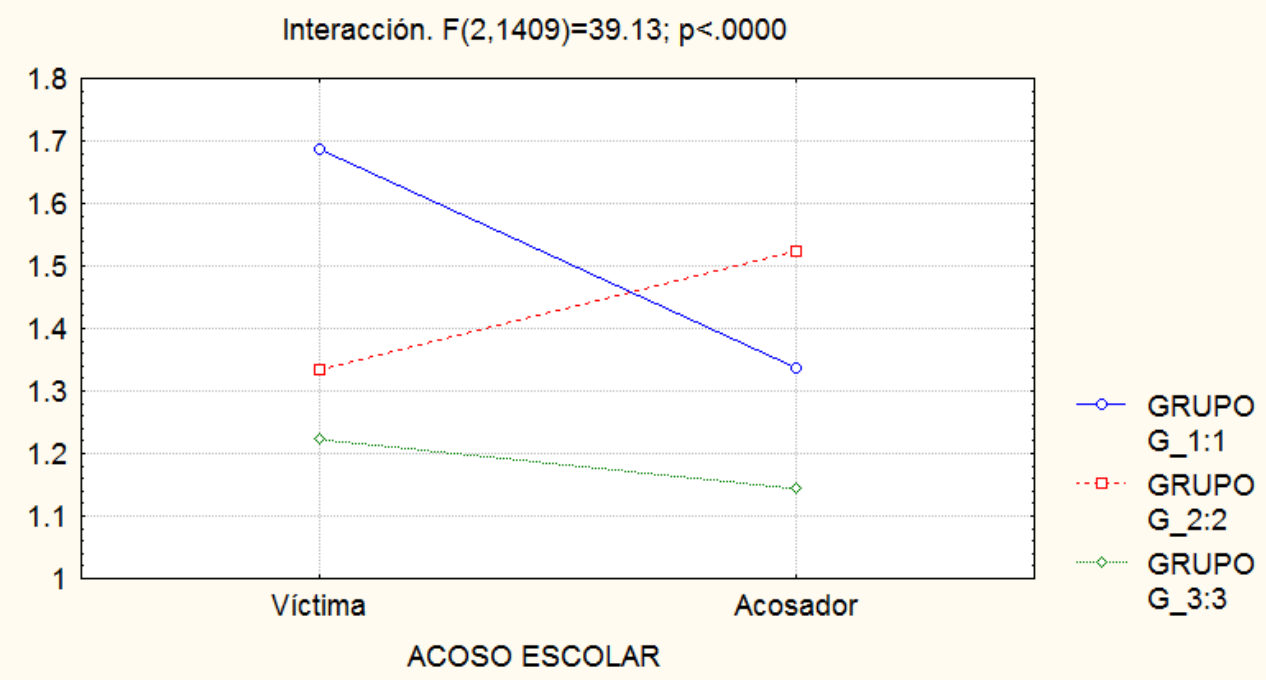

Figura 3. Interacción entre los tres grupos de personalidad y la implicación en acoso escolar como víctima o acosador -Grupo 1 = supracontrolado; grupo 2 = infracontrolado; grupo 3 = resiliente. 


\section{Diferencias según el sexo de los participantes}

En cuanto a diferencias según el sexo de los participantes en las variables estudiadas vemos en la tabla 3 que, con la prueba $t$ de comparación de medias, se confirman las más altas puntuaciones de los varones en psicoticismo $(t=10,76 ; \mathrm{p}=, 000)$ así como también las puntuaciones más altas de las mujeres en neuroticismo $(t=-6,29 ; \mathrm{p}=, 000)$, siendo las diferencias estadísticas altamente significativas. De manera similar, en agresión física $(t=$ $13,90 ; \mathrm{p}=, 000)$ y verbal $(t=4,33 ; \mathrm{p}=, 000)$ los varones informan ser superiores a las mujeres, sin diferencia de género en la subescala de hostilidad. Igualmente, los varones están más implicados que las mujeres en el maltrato escolar, tanto como víctimas o bien como maltratadores, especialmente participando en el maltrato o acoso a otros compañeros $(t=7,73$; $\mathrm{p}=, 000)$.

Tabla 3. Diferencias de sexo en las puntuaciones medias de las escalas del estudio y prueba t

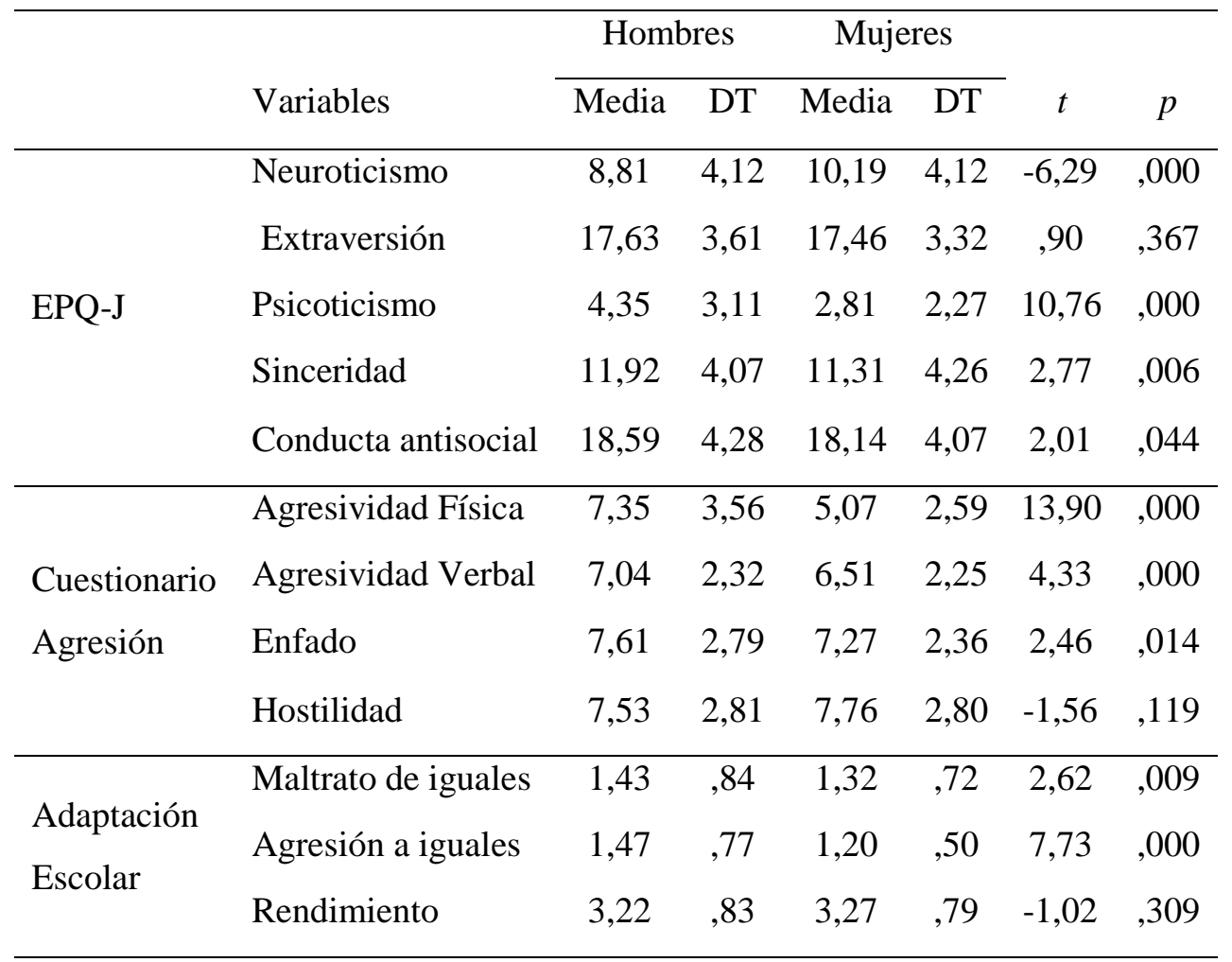

Nota. EPQ-J = Cuestionario de Personalidad de Eysenck.

Con un enfoque centrado en las variables, como los análisis de la tabla 3, hemos comprobado que ambos sexos se hallan igualados en su aprovechamiento en general en los estudios, es decir, en su rendimiento académico. Con el enfoque centrado en las personas, en cambio, ya hemos señalado la superioridad del tipo resiliente con relación a los otros dos tipos (supracontrolado e infracontrolado), con diferencias estadísticas altamente significativas (ver tabla 2) en rendimiento académico. La composición de los tipos en cuanto al sexo de sus integrantes aparece en la tabla 1. 


\section{Discusión}

La finalidad del estudio que se ha presentado ha sido analizar los tipos de personalidad y su relación con la agresividad y la conducta antisocial en una muestra de adolescentes escolarizados. En concreto, se han analizado los tipos de personalidad en relación con la hipótesis de Eysenck sobre la conducta antisocial y el nivel de agresividad.

A través de los análisis de conglomerados, con las medidas del modelo de Eysenck en el EPQ-J, hemos podido comprobar la hipótesis de la conducta antisocial. La novedad estriba en que no ha sido una comprobación con grupos formados a priori, como en el estudio de Center et al. (2005), sino con grupos formados a posteriori mediante análisis de conglomerados. Con el mismo análisis de conglomerados y el procedimiento K-medias para la solución de tres conglomerados o tipos de personalidad, el tipo infracontrolado de este estudio es del todo coincidente con el tipo infracontrolado hallado por Grumm y von Collani (2009) quienes, empleando el modelo de los Cinco Factores, caracterizaron al mismo con baja amabilidad y baja responsabilidad, las dos facetas del psicoticismo en el modelo de Eysenck. El tipo infracontrolado fue el que más puntuó en agresividad global y el resiliente el que menos, aunque la muestra no era propiamente de adolescentes. La elección del modelo de PEN de Eysenck para este estudio lo creemos plenamente justificado porque sus cuatro medidas son semejantes en muchos y muy diversos países (Barrett, Petrides, Eysenck y Eysenck, 1998). La innovación metodológica del enfoque o acercamiento centrado en las personas con este modelo ha mostrado ser una acierto por las diferencias estadísticas altamente significativas entre los tres tipos de personalidad, junto con la validación de las cuatro clases del Cuestionario de Agresión (física, verbal, enfado y hostilidad) y las tres escalas de Adaptación Escolar (dos de relación interpersonal con iguales y una de rendimiento académico). La superioridad descriptiva del acercamiento tipológico con respecto al acercamiento dimensional, así como también su utilidad, están siendo cada vez más aceptadas por los investigadores (Asendorpf, 2006). Los hallazgos de Robins et al. (1996) sitúan a los supracontrolados por debajo de los resilientes e infracontrolados en extraversión, al mismo tiempo que los resilientes se muestran superiores en estabilidad emocional (por bajo neuroticismo) a supercontrolados e infracontrolados. Estos resultados, y los obtenidos en otros estudios que también han utilizado K-medias en la formación de conglomerados, han confirmado la existencia de estos tres tipos de personalidad, junto con las diferencias características en el funcionamiento psicosocial de cada uno.

Con el modelo PEN,Connolly y O’Moore (2003) estudiaron a niños y adolescentes con edades comprendidas entre los 6 y 16 años que maltrataban a sus compañeros; de los cuales 115 eran acosadores frente a los 113 del grupo control o no acosadores. Los acosadores obtuvieron puntuaciones más altas en psicoticismo, extraversión y neuroticismo que los no acosadores. Son resultados coincidentes con los resultados obtenidos en el presente estudio, referidos allí a dimensiones del modelo PEN y en este caso al prototipo de infracontrolados logrado con el mismo modelo de personalidad.

Los resultados más recientes sugeridos por Tackett, Daoud, De Bolle y Burt (2013) también son coincidentes con los obtenidos en el presente estudio, dado que estos autores incluyen las edades comprendidas entre los 11 y 15 años. No obstante, los resultados obtenidos 
en el estudio de Tackett et al. (2013) fueron obtenidos con una muestra de 1080 participantes de edades entre los 6 y los 18 años. Pues bien, la implicación de rasgos de personalidad asociados a la conducta antisocial nos permite deducir que sus variantes de agresión física, transgresión de normas y agresión relacional se incluyen también en la comprobación de la hipótesis de la conducta antisocial de Eysenck. En los jóvenes normales se constata que la hipótesis de la conducta antisocial de Eysenck se cumple, ya se usen las medidas del Modelo PEN o bien las del Modelo de los Cinco Factores, con puntuaciones bajas en amabilidad y en responsabilidad en el prototipo infracontrolado. Como ejemplo bastante reciente, Fossati, Borroni y Maffei (2012) confirman que las puntuaciones bajas en amabilidad y en responsabilidad caracterizaban a los adolescentes que informaron conductas de maltrato.

\section{Implicaciones}

Se intenta actualmente incluir la agresión relacional como un tercer subfactor de la conducta antisocial, al lado de la agresión física y la conducta de infringir normas (Tackett et al., 2013). Estos autores aportan que los tres subfactores han mostrado pesos sustanciales en el factor general de conducta antisocial. Los hallazgos de Tackett et al. (2013), con más de mil niños y adolescentes, proporcionan soporte para el agrupamiento de la agresión relacional como subfactor de la conducta antisocial, aunque la agresión física es la variante más poderosa de la conducta antisocial juvenil, en comparación con la agresión relacional y la transgresión de normas. En cualquier caso, el estudio de la agresión relacional y su relación con la personalidad es un campo de investigación preferente para el futuro en los ámbitos educativos.

El estudio e intervención en la agresión entre iguales tendrá que implicar en sus metas el autocontrol de los estudiantes, como ha postulado la teoría del ego-control de Block y Block (1980). Ello supone caracterizar al tipo infracontrolado, tal como ya fue intentado por Block, Block y Keyes (1988), aunque sólo fuese para comprobar que ciertas respuestas de autoinforme tienen correlaciones significativas con el uso de drogas como la marihuana. Estos ítems detectan la dificultad del joven adolescente para posponer la gratificación, su reacción precipitada y rápida, su notable expresividad emocional, su labilidad emocional y su facilidad para irritarse o enfadarse ante la menor contrariedad. Según Block et al. (1988), las correlaciones de los ítems aludidos son significativos con el uso de marihuana y drogas duras, tanto para chicos como para chicas, con comienzo en la niñez. Esta deficiencia en el autocontrol está muy asociada con la dimensión psicoticismo del cuestionario EPQ-J de Eysenck, dimensión destacada en el perfil que hemos denominado infracontrolado a lo largo del presente estudio.

En cuanto a las diferencias por clases de agresión, los grupos muestran mayor propensión a la agresión física, verbal y al enfado si son infracontrolados. El prototipo de infracontrolados es el prototipo más agresivo, sólo superado en hostilidad por el grupo de supracontrolados. El prototipo resiliente no muestra predisposición agresiva alguna en comparación con los otros dos grupos o prototipos. Las diferencias de género se manifiestan en la alta distribución de mujeres entre los supracontrolados y la alta distribución de hombres entre los infracontrolados, precisamente por las altas puntuaciones en psicoticismo en su perfil, el cual es estable de los 11 a los 15 años. En contra de la crítica de Poropat (2011), los problemas de medida y validez de la dimensión psicoticismo no significan que su uso carezca de utilidad en los centros educativos. Nosotros pensamos que se ha de refinar la investigación 
con el modelo y medidas de Eysenck, porque pueden ser útiles en el psicodiagnóstico y orientación escolar, particularmente de los jóvenes adolescentes. Lo mismo piensan otros autores (e.g., Scholte y De Bruyn, 2001; López y López, 2003; Flores-Mendoza, Widaman, Mansur-Alves, Bacelar y Saldanha, 2013). De todos modos, hemos elegido el enfoque centrado en las personas, que incluye a psicoticismo dentro de un perfil clasificatorio, antes que el enfoque dimensional o centrado en las variables, quedando este último enfoque más expuesto a la crítica de Poropat (2011).

Los intentos de aproximación a la agresión relacional nos persuaden de la importancia de conocer mejor a los estudiantes más implicados, su bajo rendimiento académico y las consecuencias que afectan sobre todo a las víctimas de la agresión relacional. De acuerdo con Dahlen, Czar, Prather y Dyess (2013), la agresión relacional y la victimización correlacionan positivamente con problemas emocionales y de conducta, como problemas de depresión, ansiedad, estrés o uso de drogas. En una muestra de 2003 adolescentes de 11 a 16 años, en Irlanda del Norte, se halló que el 17,2\% había sido víctima de acoso escolar o acosados alguna vez, y el 8,1\% que había acosado a otros alguna vez. Este maltrato entre iguales, que era en realidad agresión relacional, se extendía a experiencias personales durante las horas de comer, en actividades deportivas y en la inseguridad personal percibida (Mc Guckin, Cummins y Lewis, 2009).A pesar de todo, la agresión relacional puede no aparecer como una conducta típicamente agresiva.

\section{Agradecimientos}

Estos datos forman parte de una investigación más amplia financiada por la Junta de Castilla y León (España), Resolución EDU/1160/2008, a quien agradecemos la ayuda financiera proporcionada.

\section{Referencias}

Anderson, C. A. y Bushman, B. J. (2002). Human aggression. Annual Review of Psychology, 53, 27-51.

Asendorpf, J. B. (2006). Typeness of personality profiles: A continuous person-centred approach to personality data. European Journal of Personality, 20, 83-106.

Barrett, P. T., Petrides, K. V., Eysenck, S. B. G. y Eysenck, H. J. (1998). The Eysenck Personality Questionnaire: an examination of the factorial similarity of P, E, N, and L across 34 countries. Personality and Individual Differences, 25, 805-819.

Bettencourt, B. A., Talley, A., Benjamin, A. J. y Valentine, J. (2006). Personality and aggressive behavior under provoking and neutral conditions: A meta-analytic review. Psychological Bulletin, 132(5), 751-777.

Block, J. H. y Block, J. (1980). The role of ego-control and ego-resiliency in the organization of behavior. En W. A. Collins (Ed.), Development of cognition, affect, and social relations: The Minnesota symposia on child psychology (Vol. 13, pp. 39-101). Hillsdale, NJ: Erlbaum. 
Block, J., Block, J. H. y Keyes, S. (1988). Longitudinally foretelling drug usage in adolescence: Early childhood personality and environmental precursors.Child Development, 59, 336355.

Bryant, F. B. y Smith, B. D. (2001). Refining the architecture of aggression: a measurement model for the Buss-Perry Aggression Questionnaire. Journal of Research on Personality, 35, 138-167.

Buss, A. H. (1961).The psychology of aggression.Nueva York: Wiley.

Buss, A. H. y Perry, M. P. (1992). The aggression questionnaire. Journal of Personality and Social Psychology, 63, 452-459.

Center, D., Jackson, N. y Kemp, D. (2005). A test of Eysenck's antisocial behavior hypothesis employing 11-15-year-old students dichotomous for PEN and L. Personality and Individual Differences, 38, 395-402.

Connolly, I. y O’Moore, M. (2003). Personality and family relations of children who bully. Personality and Individual Differences, 35, 559-567.

Crick, N. R. y Dodge, K. A. (1996). Social information-processing mechanisms in reactive and proactive aggression. Child Development, 67, 993-1002.

Dahlen, E. R., Czar, K. A., Prather, E. y Dyess, C. (2013). Relational aggression and victimization in college students. Journal of College Student Development, 54, 140-154.

Dodge, K. A. y Coie, J. D. (1987). Social-information-processing factors in reactive and proactive aggression in children`s peer groups. Journal of Personality and Social Psychology, 53, 1146-1158.

Egan, V.(2009). The "Big Five": Neuroticism, Extraversion, Openness, Agreeableness, and Conscientiousness as an organization scheme for thinking about aggression and violence. En M. McMurran y R. Howards (Eds.), Personality, personality disorder, and risk of violence: An evidence-based approach (pp. 63-84). Chichester, UK: John Wiley y Sons.

Eklund, J. M., Liljeberg, J. F. y Klinteberg, B. af (2011). Delinquent behavior patterns in adolescence: Development and associated personality traits. Personality and Mental Health, 5, 169-185.

Eysenck, H. J. (1992). A reply to Costa and McCrae.P or A, and C - the role of theory.Personality and Individual Differences, 13, 867-868.

Eysenck, H. J. (1997). Personality and the biosocial model of anti-social and criminal behavior. En A. Raine, P. Brennan, D. Farrington y S. Mednick (Eds.). Biosocial bases of violence (pp. 21-37). Nueva York: Plenum.

Eysenck, H. J. y Eysenck, M. W. (1985). Personality and individual differences.Nueva York: Plenum.

Eysenck, H. J. y Eysenck, S. B. G. (1998). EPQ - A y J. Cuestionario de Personalidad para Niños y Adultos. Madrid: TEA Ediciones.

Eysenck, S. B. G., Eysenck, H. J. y Barrett, P. (1985). A revised version of the Psychoticism scale. Personality and Individual Differences, 6, 21-29.

Fossati, A., Borroni, S. y Maffei, C. (2012). Bullying as a style of personal relating: Personality characteristics and interpersonal aspects of self-reports of bullying behaviors among Italian high school students. Personality and Mental Health, 6, 325-339. 
Flores-Mendoza, C., Widaman, K., Mansur-Alves, M., Bacelar, T. D. y Saldanha, R. (2013). Psychoticism and disruptive behavior can be also good predictors of school achievement. The Spanish Journal of Psychology, 16 (January).

Griffin, R. S. y Gross, A. M. (2004). Childhood bullying: Current empirical findings and future directions for research. Aggression and Violent Behavior, 9, 379-400.

Grumm, M. y von Collani, G. (2009). Personality types and self-reported aggressiveness. Personality and Individual Differences, 47, 845-850.

Klimstra, T. A., Hale III, W. W., Raaijmakers, Q. A. V., Branje, S. J. T. y Meeus, W. H. J. (2010). A developmental typology of adolescent personality. European Journal of Personality, 24, 309-323.

López, C. y López, J. R. (2003). Rasgos de personalidad y conducta antisocial y delictiva. Psicopatología Clínica Legal y Forense, 3, 5-19.

Mc Guckin, C., Cummins, P. K. y Lewis, C. A. (2009). Bully/victim problems in Northern Ireland's schools: data from the 2003 young persons' behaviour and attitude survey. Adolescence, 44, 347-358.

Mendoza, B., Morales, T. y Arriaga, Y. (2015). Variables proximales relacionados con violencia escolar y bullying en alumnado de bachillerato. Psychology, Society \& Education, 7, 185-200.

Ojanen, T., Findley, D. y Fuller, S. (2012). Physical and relational aggression in early adolescence: Associations with narcissism, temperament, and social goals. Aggressive Behavior, 38(2), 99-107.

Ortega, R., Elipe, P. y Calmaestra, J. (2009). Emociones y agresores y víctimas de cyberbullying: Un estudio preliminar en estudiantes de secundaria. Ansiedad y Estrés, 15(2-3), 151-165.

Poropat, A. E. (2011). The Eysenckian personality factors and their correlations with academic performance. British Journal of Educational Psychology, 81(1), 41-58.

Ramírez, M. Díaz, F. y López, R. (2015). Maltrato entre escolares: Diagnóstico en estudiantes de nivel secundaria en la ciudad de Méjico. Psychology, Society \& Education, 7, 169184.

Robins, S. W., John, O., Caspi, A., Moffit, A. y Stouthamer-Loeber, M. (1996). Resilient, overcontrolled, and undercontrolled boys: Three replicable personality types. Journal of Personality and Social Psychology, 70, 157-171.

Roseth, C. J., Johnson, D. W. y Johnson, R. T. (2008). Promoting early adolescents' achievement and peer relationships: The effects of cooperative, competitive, and individualistic goal structures. Psychological Bulletin, 134, 223-246.

Sánchez, V., Ortega, R. y Menesini, E. (2012). La competencia emocional de agresores y víctimas de bullying. Anales de Psicología, 28, 71-82.

Scholte, R. H. J. y De Bruyn, E. E. J. (2001). The Revised Junior Eysenck Personality Questionnaire (JEPQ-R): Dutch replications of the full-length, short, and abbreviated forms. Personality and Individual Differences, 31, 615-625.

Tackett, J. L., Daoud, S. L. S. B, De Bolle, M. y Burt, S. A. (2013). Is relational aggression part of the externalizing spectrum? A bifactor model of youth antisocial behavior. Aggressive Behavior, 39(2), 149-159. 
Viding, E., Fontaine, N. M. y McCrory, E. J. (2012). Antisocial behavior in children with and without callous-unemotional traits. Journal of the Royal Society of Medicine, 105(5), 195-200.

Viding, E., Sinmonds, E., Petrides, K. y Frederickson, N. (2009). The contribution of callousunemotional traits and conduct problems to bullying in early adolescence. Journal of Child Psychology and Psychiatry, 50(4), 471-481. 